\title{
La noción de transferencia política en el marco de la historia transnacional: una propuesta de conceptualización
}

\author{
The notion of political transference in the framework of \\ transnational history: A proposal of conceptualization
}

Joaquín Fernández Abara*

\section{RESUMEN}

El artículo propone una definición del concepto de transferencia política, en gran medida tributaria de la conceptualización realizada por el historiador Henk Te Velde. Se sostiene que dicha noción aporta una perspectiva de análisis útil para el estudio de los fenómenos políticos, principalmente ideas y prácticas, que trascienden las fronteras nacionales en contextos en que los Estados nacionales son entidades operantes relevantes. Con dicho fin, se sitúa a la noción de transferencia en el marco de la historia transnacional; se señalan los aportes que pueden incorporarse de los estudios de circulación de las ideas, policy transfer y sociología de la difusión, $\mathrm{y}$, finalmente, se reflexiona sobre el rol de los agentes en dichos procesos. El texto está fundamentado en una investigación de carácter bibliográfico, principalmente a través del uso de textos de reflexión historiográfica, sociológicos y politológicos.

\section{SUMMARY}

This article proposes a definition of the concept political transference, predominantly affluent to the conceptualization made by historian Henk Te Velde. We argue that this notion provides a useful analytical perspective for studying political phenomena, main ideas, and practices that transcend national borders in contexts where nation-states are relevant operating entities. The notion of transference is placed within the framework of transna-
Palabras clave: transferencia política, historia transnacional, transferencia cultural, modelos políticos.
Keywords: Political transference, transnational history, cultural transference, political models

\footnotetext{
* Chileno. Candidato a Doctor en Historia, Universidad de Leiden, Países Bajos. Profesor investigador, Centro de Investigación y Documentación (CIDOC) y Escuela de Historia, Universidad Finis Terrae, Chile. E-mail: jfernandez@uft.cl
} 
La noción de transferencia política en el marco de la historia transnacional | Fernández

tional history. We point out contributions incorporated from the circulation of ideas, policy transfer, sociology of diffusion, and these processes agents' role. This article stands on bibliographic research, mainly through historiographic, sociological, and political texts. 
El estudio de la historia política se ha visto fuertemente influenciado por la inclusión de las perspectivas global y transnacional. Más allá de la preocupación por las relaciones internacionales, la necesidad de dar cuenta del carácter global de los fenómenos políticos y de cómo muchos de estos trascienden las fronteras nacionales, ha implicado que la historiografía deba adaptar sus conceptos, enfoques y metodologías. Sin embargo, cabe preguntarse ¿cómo estudiar fenómenos que trascienden las fronteras nacionales en contextos en que los estados nacionales son entidades operantes relevantes? ¿Como se generan los modelos y contramodelos políticos en dichas circunstancias?

Para responder dichas preguntas, en el presente artículo conceptualizaremos la noción de transferencia política basándonos en la definición de Henk Te Velde (2005). En este sentido, consideraremos a la transferencia política como la migración, a través de las fronteras nacionales, de prácticas, representaciones e ideas políticas, además de modelos de políticas ya implementados. Planteamos que dicha conceptualización permite generar una perspectiva de análisis adecuada para estudiar los fenómenos políticos que trascienden las fronteras nacionales y circulan a través de ellas, en un contexto en que estas son, de todas maneras, entidades operantes relevantes. Del mismo modo, la definición recién expuesta permite al investigador contar con una perspectiva de análisis adecuada para entender la génesis y el desarrollo de modelos y contramodelos políticos de carácter transnacional. Con este fin, situaremos a la noción de transferencia en el contexto de los debates recientes sobre la historia transnacional, indicaremos los enfoques de distintas disciplinas de los que dicha perspectiva se puede nutrir, y señalaremos el modo en que permite abordar la relación entre la agencia de los actores involucrados y los constreñimientos impuestos por las instituciones y las tradiciones. De acuerdo con dichos objetivos, el texto ha sido dividido en tres secciones, ordenadas según un criterio temático en una progresión desde aspectos generales a particulares.

En la primera sección, titulada "El giro transnacional en la historiografía", abordaremos algunas de las transformaciones que ha vivido la historiografía en las últimas tres décadas, centrándonos en la tendencia a relativizar y aquilatar la importancia de los Estados nacionales como escala de análisis. Con este fin, señalaremos las transfor- 
maciones en las corrientes intelectuales que han hecho posible dicho cambio y las condiciones de producción en que estas han tenido lugar. Profundizaremos en los enfoques historiográficos que han emergido producto de estas transformaciones, como son la historia global y la historia transnacional, señalando cómo esta última es la más apropiada para enmarcar la conceptualización que vamos a generar. De esta manera, podemos situar nuestra contextualización en una perspectiva funcional al estudio de fenómenos que trascienden y desbordan a los Estados nacionales y sus fronteras, teniendo en cuenta la existencia de estos y su importancia como fuerza operante.

En la segunda sección, titulada "El estudio de la transferencia política", desarrollaremos una definición del concepto de transferencia política. Nuestro principal referente será la conceptualización del historiador neerlandés Henk Te Velde. Para cumplir dicho propósito, nos apoyaremos en los estudios sobre transferencia cultural, señalando las características de estos, en diálogo con otros enfoques metodológicos relacionales en el marco de la historia transnacional, como son la historia comparativa y la histoire croisée. También señalaremos algunos aportes que pueden servir para complementar y enriquecer dicho concepto, los que provienen desde enfoques metodológicos de distintas disciplinas que han tenido escaso contacto entre sí, como son los estudios historiográficos y literarios de circulación de las ideas y de recepción, y los estudios politológicos de policy transfer. De este modo, podemos tener en cuenta las prevenciones aportadas por dichos enfoques, con tradiciones portadoras de una significativa experiencia de estudios con importantes similitudes y puntos de contacto.

En la tercera sección, titulada "El rol de los agentes en el proceso de transferencia política", identificaremos y tipologizaremos a los actores que llevan adelante el proceso de transferencia, para luego analizar su relación con el contexto en que se desenvuelven, los constreñimientos que operan sobre ellos y su capacidad para generar cambios en su entorno. Con dicho fin, recurriremos a un arsenal conceptual enriquecido gracias a elementos de la sociología de la difusión. De esta manera, podemos ponderar la importancia de los elementos estructurales - en un sentido amplio- y agenciales en la creación de modelos y contramodelos políticos en una perspectiva transnacional. 
Para lograr nuestro cometido, hemos recurrido a un corpus bibliográfico proveniente de distintas diciplinas. Así, hemos considerado textos que se han centrado en analizar los debates historiográficos actuales, enfatizando en las perspectivas global y transnacional. También hemos considerado obras de sociología, principalmente aquellas que han estudiado procesos de difusión de formas de acción colectiva; politología, especialmente aquellas que han estudiado cómo se transfieren las políticas públicas, y textos sobre circulación de las ideas y recepción. De esta manera hemos tomado la opción explícita de complementar las reflexiones historiográficas con las partes de disciplinas que han estudiado problemas similares y funcionales a nuestros propósitos.

\section{El giro transnacional en la historiografía}

Desde fines de la década de 1980 ha tenido lugar un importante proceso de reorientación en las corrientes historiográficas, el que ha sido conocido con diversos nombres, como los de giro global y giro transnacional. Al respecto, consideramos necesario introducir brevemente las transformaciones vividas por el campo historiográfico y su contexto, las que han hecho posible la reorientación de los intereses de estudio hacia los ámbitos global y transnacional. Cabe señalar que no necesariamente adherimos a todas las concepciones y presupuestos que vamos a dar a conocer en esta primera sección, lo que se evidenciará tanto en el momento de explicitar nuestra opción por el concepto de transferencia política como cuando lo definamos. Sin embargo, consideramos necesario comenzar dando cuenta de algunos de los debates y problemas que han hecho posible este cambio en la historiografía para luego posicionar ante ellos de manera más clara nuestra conceptualización de la noción de transferencia política.

El interés por los ámbitos globales y transnacionales no es completamente nuevo y, en distintos periodos, muchas obras historiográficas se habían preocupado de trascender los límites impuestos por las fronteras nacionales en sus narrativas. Sin embargo, los últimos treinta años se han caracterizado por la creciente importancia y centralidad que el afán de superación de los marcos nacionales ha tenido en el quehacer historiográfico y por la aparición de gran cantidad de obras de reflexión disciplinar destinadas a tematizar este tópico. Como han señalado Bernhard Struck, Kate Ferris y Jacques Revel, 
"desde los años 90 tempranos, y aún más significativamente desde inicios de la década del 2000, la creciente frecuencia del uso del término historia transnacional - junto con el de historia globalindican que algo está sucediendo en la historia y sus disciplinas aledañas" (Struck, at al., 2011: 573). ${ }^{1}$

Pese a la existencia de diversas denominaciones para estas nuevas aproximaciones a la historia, las que dejan entrever un amplio y variopinto abanico de intereses investigativos, bases teóricas, objetivos, metodologías y fuentes, todas comparten el "interés en que los marcos de la narración" y el análisis histórico "excedan los de la nación" (Balachandran, 2015: 533).

El contexto en que ha tenido lugar la producción historiográfica ha jugado un papel fundamental en esta reorientación de los intereses de investigación disciplinares. Al respecto, cabe tener en cuenta los importantes cambios sucedidos en las últimas décadas, en especial desde fines de los años ochenta, en los ámbitos político, económico, sociocultural e intelectual, y cómo estos han coadyuvado en la transformación de los intereses investigativos.

Algunos de los cambios sociales generales acaecidos en las últimas décadas han tendido a debilitar a los Estados nacionales. Dentro de estas transformaciones, podemos encontrar fenómenos tan diversos como la caída del bloque de países socialistas; la creciente interdependencia económica planetaria; el auge de corporaciones empresariales multi y transnacionales; el deterioro de la capacidad de los Estados para regular y controlar la economía, con el consiguiente retroceso de las tendencias proteccionistas que habían caracterizado la segunda mitad del siglo XX, y la aceleración y complejización de los medios de comunicación y las tecnologías de la información, entre otros. Existe un amplio debate en las ciencias sociales y las humanidades sobre los contenidos y los alcances del término globalización (Osterhammel y Petterson, 2019: 9-18). Sin embargo, los fenómenos recién nombrados tienden a estar incluidos dentro de los análisis que han dado pie a la emergencia de dicho concepto.

Todas las citas de textos en otro idioma original son traducción propia. 
No es de extrañar que, en este contexto, los historiadores hayan resaltado la historicidad del Estado nacional, a la vez que han tomado conciencia de sus limitaciones, generando un cambio en la reflexividad disciplinar. De este modo, como sostienen Werner y Zimmermann, la preocupación por el problema de la globalización "ha dejado una marca en los paradigmas investigativos, dando una nueva importancia al problema de la reflexividad" (Werner y Zimmermann, 2006: 30). Incluso, la gran mayoría de los estudios sobre nacionalismo e identidades nacionales, que también han vivido un "revival" en estas décadas, se han centrado en el carácter histórico, contingente e incluso moderno de dichos fenómenos (Anderson, 1983; Hobsbawm, 1992 y Gellner, 1983). ${ }^{2}$

Fuera del contexto sociohistórico general en que ha tenido lugar la producción historiográfica en las últimas décadas, cabe mencionar los cambios vividos al interior de los propios espacios de producción del conocimiento historiográfico. Tras los procesos de profesionalización y especialización disciplinar, que tuvieron lugar en Europa y Estados Unidos en el siglo XIX, y en América Latina en el siglo XX, el espacio fundamental para el desarrollo de la disciplina historiográfica ha sido la universidad. Si bien las pretensiones universalistas siempre han estado presentes en la academia, en los últimos años estas aspiraciones se han visto facilitadas en el trabajo académico cotidiano. El creciente intercambio académico, la vinculación con instituciones internacionales de cooperación y la mayor facilidad en el acceso a la producción académica generada en distintas latitudes gracias a las tecnologías de la información, han contribuido a internacionalizar las instituciones universitarias y las prácticas académicas, al punto que la internacionalización se ha vuelto una meta explícita de muchas instituciones académicas (Struck et al., 2011: 575). Esta situación ha propiciado la emergencia de nuevos intereses de investigación que superan los mar-

\footnotetext{
2 Una síntesis clara y completa referida a los debates teóricos sobre el nacionalismo, puede ser encontrada en Smith (2008). Tras un balance provisional, se puede afirmar que, en la actualidad, existe una hegemonía de las tesis modernistas sobre el origen y el desarrollo de los nacionalismos y las identidades nacionales en los medios académicos. Con estos nos referimos a los planteamientos que sostienen que el proceso de emergencia de las identidades nacionales es un fenómeno de carácter moderno. Un contrapunto a dichas interpretaciones, que refuta las tesis modernistas, ha sido realizado por Hastings (1997). Para una síntesis crítica de los planeamientos de Hastings, véase Fernández Abara (2005).
} 
cos nacionales, al mismo tiempo que ha facilitado la posibilidad de llevarlos a cabo.

Además de cambios contextuales en las condiciones de producción intelectual que ya hemos mencionado, y en directa relación con ellos, es necesario tener en cuenta cómo algunos de los cambios en las preocupaciones y paradigmas de investigación de las humanidades y las ciencias sociales, que han tenido lugar en las últimas décadas, han estimulado indirectamente la emergencia de los giros global y transnacional en la discusión historiográfica.

Con esto nos referimos, en primer lugar, al surgimiento de corrientes de reflexión en las ciencias sociales que se han mostrado críticas del nacionalismo metodológico. Como ha señalado Daniel Chernilo, quien ha generado una definición operativa para dicho concepto, el nacionalismo metodológico sería la "igualación entre el estado-nacional y la sociedad", en que la que el "estado nacional pasaría ser la forma natural, necesaria e incluso automática de sociedad en la modernidad" (Chernilo, 2007: 209). Según Chernilo, en las últimas cuatro décadas han surgido diversas corrientes que han criticado la tendencia a igualar los conceptos de "Estado nacional" y "sociedad". Para el autor, algunas de estas críticas se habrían planteado desde una perspectiva lógica, que sostenía que el nacionalismo metodológico se habría generado como el producto del desarrollo interno de las tendencias intelectuales y de las prácticas institucionales de la sociología. Mientras que otra vertiente habría formulado críticas desde un punto de vista histórico, sosteniendo que el nacionalismo metodológico de la sociología tendría sus orígenes en la fuerza que tuvo el nacionalismo como ideología de Estado durante el siglo XX. Chernilo también ha sostenido que, más recientemente, en la década del 2000, han surgido nuevas críticas al nacionalismo metodológico, las que apuntarían a que este sería un factor que impediría un conocimiento acabado de la sociedad en el marco de las transformaciones generadas por la globalización (Chernilo, 2006). ${ }^{3}$

Cabe aclarar que Chernilo, si bien manifiesta una postura opuesta al nacionalismo metodológico, ha expresado objeciones al modo en que, hasta el momento, se han planteado las críticas sobre este, ya que, a pesar de rechazarlo, habrían sido incapaces de trascenderlo (Chernilo, 2006: 6). 
Además de las críticas al nacionalismo metodológico, es importante tener en cuenta, en segundo lugar, otro cambio en las corrientes historiográficas y sus disciplinas afines. Nos referimos al cultural turn o giro cultural, que ha reforzado las aproximaciones hacia lo global y lo transnacional. El giro cultural ha tendido a descentrar la mirada de los factores institucionales y de las estructura y procesos sociales, para pasar a prestar atención a elementos de índole cultural en la narración historiográfica. En este sentido, la creciente importancia de la antropología cultural y la apropiación de muchos de sus elementos por parte de la historiografía ha jugado un papel fundamental (Burke, 1999: 80-89). Dentro de estas nuevas corrientes, han adquirido especial fuerza visiones que tienden a privilegiar la importancia del lenguaje y la comunicación, dando pie a la emergencia del giro lingüistico en la disciplina. Este panorama ha sido descrito por Georg Iggers, quien ha señalado que:

“El elemento central de este 'giro' consiste en el reconocimiento de la importancia del lenguaje o discurso en la constitución de las sociedades: Las estructuras o procesos sociales, que eran vistas como determinantes de una sociedad y cultura, son cada vez más entendidas como producto de la cultura en tanto comunidad comunicativa" (Iggers, 2012: 200).

En el contexto de estas transformaciones, no es extraño que las unidades de análisis en que se enfocaba el trabajo historiográfico hayan pasado de los Estados nacionales a diversas formas de comunidad e identidad, las que pueden desdibujar la centralidad de las fronteras nacionales en las narrativas. Dicha situación se ve reforzada por la importancia que han adquirido las diversas formas de circulación y transferencia cultural en los análisis culturalistas.

A la crítica al nacionalismo metodológico y a los énfasis culturalistas se ha sumado, en tercer lugar, la irrupción en los circuitos académicos internacionales de comunidades académicas provenientes de países asiáticos, africanos y latinoamericanos, muchos de ellos marcados por su historia colonial, los que han dado pie al surgimiento de corrientes que cuestionan la universalización de las categorías de análisis de origen occidental para conocer la historia de sus regiones de origen. Una de las principales expresiones de estas corrientes, los estudios subalternos, han cuestionado los enfoques centrados en el estudio del Estado- 
nación, llegando incluso a considerarlos como una forma de imperialismo cultural y poniendo en duda su capacidad para dar cuenta de las complejidades de su historia (Iggers, 2012: 243; Chatterjee, 2001: 15237-15241). Este planteamiento ha sido rescatado por comunidades académicas centradas en el estudio y la reivindicación identitaria de minorías raciales y étnicas, en diversas latitudes, quienes, también, han adoptado sus críticas a la centralidad del Estado-nacional (Mantra, 2005: 161-166). Así, los debates sobre el multiculturalismo, que han tenido gran resonancia en el espacio público, también han influido en la academia y, más específicamente, en la historiografía. Del mismo modo, en las antiguas potencias coloniales los historiadores han comenzado a ocuparse de los efectos que su propio pasado colonial tuvo en su desarrollo histórico, enfatizando en el carácter global e interconectado de su historia (Conrad, 2009: 52-77). ${ }^{4}$

Así pues, existe una amplia gama de factores contextuales y orientaciones intelectuales que han incidido en los afanes por trascender las fronteras nacionales en el análisis y en la narración historiográfica. Muchos de estos son distintos e incluso algunos pueden llegar a veces a ser contradictorios entre sí. Quizás esto explica la ausencia de una denominación unívoca para las nuevas perspectivas que se han generado gracias a estas corrientes renovadoras.

En este sentido, se vuelve necesario realizar un esfuerzo para conocer las principales denominaciones que han recibido estas nuevas orientaciones en la historiografía. Al respecto, consideramos necesario realizar una distinción entre los conceptos de historia global e historia transnacional, dos de los más utilizados para describir estas transformaciones. Si bien no existen textos canónicos que nos permitan adherir a una definición exacta preexistente, sí podemos generar distinciones entre los dos conceptos, basándonos en textos de sus cultores y en algunos autores que han intentado generar un debate en torno a estas categorías.

\footnotetext{
4 Sebastian Conrad, quien ha estudiado un tema escasamente abordado, como es el de la experiencia colonial alemana, sostiene que "la emergencia y el desarrollo de sociedades modernas en Europa se encuentra ligado de manera constitutiva a sus intervenciones coloniales" (Conrad, 2009: 52-77). En una lógica similar, los pensadores decoloniales latinoamericanos han planteado que la colonialidad sería parte integral de los propios proyectos modernizadores, siendo esta una suerte de lado oscuro de la primera, que le es constitutivo (Maldonado-Torres, 2007: 132 y Mignolo, 1995).
} 
Al momento de intentar conceptualizar el término historia global, nos enfrentamos a la dificultad generada por su carácter polisémico. La amplia gama de autores que lo han invocado para describir sus investigaciones, lleva a que este haya servido para caracterizar los más diversos tipos de investigaciones. Así, el historiador Jean de Vries (2013) señala que el concepto de "historia global se nos presenta como una casa con muchas mansiones", argumentando que en distintos niveles puede ser invocado para caracterizar fenómenos muy diversos. Por una parte, en un nivel superior, sirve para dar cuenta de la "historia de la globalización y los megaprocesos de integración global”. Por otra, en un nivel intermedio, serviría para ofrecer un espacio de investigación para el estudio de "mundos pequeños, cuyos límites usualmente se sobreponen a través de las fronteras políticas tradicionales". Finalmente, en un último nivel, "serviría para agrupar los estudios enfocados en interacciones transnacionales de alcances y escalas modestos" (De Vries, 2013). En este sentido, dada la diversidad de enfoques y objetos de estudio que puede englobar, estamos de acuerdo con las prevenciones de Andrés Baeza, quien ha sostenido que:

"En la actualidad existe una gran tentación de nombrar cualquier cosa que conecte dos regiones distintas con la etiqueta de global. Quizás esta situación tiene lugar porque en la actualidad se ha puesto de moda hablar sobre la globalización, y porque también estamos viviendo un mundo cada vez más interconectado. De hecho, muchos historiadores están buscando en el pasado diferentes patrones y formas de vinculación con el fin de demostrar que el mundo ha estado interconectado mucho antes del estallido de la revolución tecnológica" (Baeza, 2015: 2). ${ }^{5}$

Pese al carácter polisémico del término, han existido importantes esfuerzos por definir un concepto de historia global. Al momento de realizar un ejercicio de conceptualización, Bruce Mazlish (1993: 5) sostiene que, antes que nada, es una "perspectiva" que ayudaría a orientar

En un sentido similar, Lynn Hunt ha sostenido que gran parte del corpus de obras historiográficas que se ha reconocido como "historia global es en realidad transnacional (referida a los vínculos establecidos entre dos o más lugares que no están en el mismo estado) o comparativa (comparando dos o más lugares) antes que verdaderamente global" (Hunt, 2014: 63). 
la investigación histórica, con algunas características que la harían distintiva. Para Mazlish:

"Una definición preliminar de historia global incluye varios factores. Desde un punto de vista negativo, no es eurocéntrica, ni está centrada en el estado nacional, ni en una entidad unívoca lineal y progresiva de estilo Whig. Desde un punto de vista positivo, ella emerge desde los factores de globalización existentes - considerándolos como novedosos al menos en el grado en que se han dado- y en sus interacciones; se centra en nuevos actores de variada índole; se preocupa con especial atención de la dialéctica entre lo global y lo local (por ejemplo, reconociendo que lo global usualmente ayuda a la generación de respuestas localistas); acepta métodos analíticos y narrativos que sean apropiados para el objeto de estudio del que se está ocupando y necesariamente confía en la investigación interdisciplinaria y grupal" (Mazlish, 1993: 5-6).

Cabe detenerse a observar la terminología utilizada por Mazlish. Pese a ser una conceptualización realizada de manera temprana, a inicios de la década de 1990, logra establecer límites y se plantea una agenda de investigación, lo que nos permite extraer de ella una definición mínima propia, que podemos complementar con algunos aportes de las definiciones posteriores que hemos abordado. ${ }^{6}$

Consideramos a la historia global como una perspectiva de análisis historiográfico, que busca estudiar la historia teniendo en cuenta procesos de vinculación e interconexión - tanto de índole cooperativa como conflictiva - entre actores de diverso tipo, con el fin de hacer inteligibles los procesos globalizadores de largo alcance desde una perspectiva histórica, en análisis amplios o de casos específicos. En este

\footnotetext{
$6 \quad \mathrm{Al}$ respecto, cabe tener en cuenta los planteamientos del historiador Christopher Bayly, cultor de la historia global, estudioso de la historia del Imperio británico y del Sudeste asiático, quien ha sostenido que el término "historia transnacional" resultaría "restrictivo para el tipo de trabajo en que él está centrado. Esto en cuanto, antes de 1850, grandes partes del globo no se encontraban dominadas por naciones y sí por imperios, ciudades-estados, diásporas, etc.." A esto añade que, si bien efectivamente existía "cierto sentido de nacionalidad en algunas partes del mundo no occidental", designar a la "historia global" como "historia transnacional no sería muy útil antes de 1914" (Bayly et al., 2006: 1443). Del mismo modo, Andrés Baeza, basado en planteamientos de John Darwin, ha sostenido que "gran parte de lo que en la actualidad llamamos 'historia global' es derivativa de la anteriormente llamada 'historia imperial"' (Baeza, 2015: 4).
} 
sentido, uno de los aspectos que la distingue es el constante afán de descentrar su mirada del Estado nacional y ponerlo en actores no estatales o en los procesos mismos de interconexión. No es de extrañar que esta perspectiva resulte operativa y sea especialmente atractiva para aquellos investigadores que han pretendido estudiar actores no estatales y a los que han pretendido estudiar regiones y periodos históricos en los que el Estado nacional no ha sido una forma de organización política hegemónica ni un generador de identidad relevante.

Como hemos señalado, existen determinados problemas de estudio y contextos en los cuales la historia global puede ser una perspectiva de análisis apropiada. Sin embargo, consideramos que dicha perspectiva no necesariamente es funcional al estudio de todo tipo de fenómeno que trascienda las fronteras nacionales. Esto se debería a dos motivos fundamentales. En primer lugar, porque dichos estudios no necesariamente aspiran a conocer procesos globalizadores de largo alcance, teniendo pretensiones más acotadas. Como ha sostenido Sven Beckert, mucha de la historiografía que pretende trascender las fronteras nacionales "no necesariamente tiene un enfoque global, y, por el contrario, examina regiones particulares - que pueden o no ser contiguas- conectadas entre sí por redes particulares" (Bayly et al., 2006: 1443). En segundo lugar, porque, como hemos señalado, el enfoque de historia global tiende a relegar a un rol secundario la importancia de los estados nacionales, tanto en cuanto actores, como en cuanto arenas de lucha y otras formas de interacción en que se desenvuelven los actores. Esto transformaría a la historia global en un enfoque poco apropiado para estudiar periodos en que el Estado nacional sí ha tenido un rol activo, constriñendo las posibilidades y los objetivos de los actores que estamos estudiando, aunque estos desarrollen su actividad transcendiendo sus fronteras. En este sentido, Struck, Ferris y Revel han señalado que:

"parte del mundo transnacional es nacional y la mayoría de los académicos que defienden perspectivas transnacionales no le restan relevancia a la nación. Por el contrario, la mayoría de ellos reconoce explícitamente el importante impacto que las naciones y las sociedades nacionales han tenido en las vidas de la gente y en las sociedades durante el período moderno o tardo-moderno" (Struck et al., 2011: 576). 
No es de extrañar que estos autores utilicen el término de historia transnacional señalando algunas de las categorías que pretenderían dar a dicha perspectiva de análisis. Para ellos, la historia transnacional buscaría:

"añadir nuevas perspectivas a los niveles nacional y espacial, que hayan impactado en la formación de las naciones, los estados nacionales y las vidas individuales en el pasado. Como perspectiva, la historia transnacional asume que la nación y los estados nacionales son una dimensión espacial -la que por supuesto es crucial-, pero sólo una entre otras, que van desde la historia global y las dinámicas internacionales hasta los niveles suprarregional o subnacional, local e individual" (Struck et al., 2011: 576).

Así, definimos a la historia transnacional como una perspectiva de análisis historiográfico que busca estudiar la historia teniendo en cuenta procesos de vinculación e interconexión - tanto de índole cooperativa como conflictiva- entre actores de diverso tipo, más allá de las fronteras nacionales, tomando en cuenta también la presencia y la relevancia de los Estados nacionales, considerados tanto en escenarios como en cuanto actores, que constriñen las posibilidades y los objetivos de los actores estudiados. ${ }^{7}$ Dicha perspectiva permite enriquecer las historias nacionales, señalando cómo estas se encuentran marcadas por la interacción con actores de la más diversas procedencias, cuestionando las narrativas nacionalistas, que se centran de manera fundamental en elementos endógenos. Consideramos que esta perspectiva puede resultar operativa para aquellos investigadores que estudian procesos de vinculación que trascienden las fronteras en momentos históricos y regiones en los cuales los estados nacionales se encuentran consolidados, y en que las identidades nacionales son una forma de identidad activa y hegemónica.

En este sentido, la noción de transferencia política que desarrollaremos se sitúa en el marco de la historia transnacional y se encuentra

Jürgen Osterhammel, planteándose la posibilidad de definir un proyecto y programa de investigación para una historia social transnacional, ha planteado que "dar cuenta de las virtudes de la historia social transnacional no implica una denigración general del estado nacional como unidad de análisis. El estado nacional continúa siendo el marco institucional más importante para las vidas de la mayor parte de las personas en el mundo" (Osterhammel, 2009: 47). 
pensada como un enfoque apropiado para el estudio de realidades en que los Estados nacionales son entidades relevantes. En el ámbito temporal, esto circunscribe su aplicación a periodos más bien contemporáneos, aunque relativamente variables en distintas regiones del globo.

\section{El estudio de la transferencia política}

Una vez que hemos conceptualizado la noción de historia transnacional, distinguiéndola de la historia global, es necesario señalar los principales enfoques metodológicos que se ubican en su interior, dentro de los cuales se encuentran los estudios de transferencia política. ${ }^{8}$ En este sentido, identificaremos y distinguiremos tres formas de hacer historia, todas ellas relacionadas entre sí pero con importantes diferencias; estas son la historia comparativa, la historia cruzada y la historia de las transferencias, para finalmente situarnos en este último enfoque ${ }^{9}$.

Es necesario partir por explicar los enfoques de historia comparativa, teniendo en cuenta los retos que plantea a la investigación historiográfica, puesto que los estudios de las transferencias nacieron, en parte, como una respuesta a las falencias y desafíos que estos planteaban a la investigación historiográfica. Jurgen Kocka y Heinz Gerhard Haupt sostienen que "en la Historia Comparativa, dos o más fenómenos históricos son estudiados sistemáticamente en sus similitudes y diferencias, contribuyendo a mejorar su descripción, explicación e interpretación" (Kocka y Haupt, 2009: 2). Para los autores, quienes en este aspecto siguen los ya clásicos planteamientos de Otto Hintze, el ejercicio comparativo puede tener dos enfoques, de acuerdo a sus objetivos: estos pueden ser generalizadores, buscando aspectos en común, o individualizantes, pretendiendo destacar cualidades particulares de los objetos de estudio. Si bien coinciden en que dicha distinción es fundamental, plantean que todo ejercicio comparativo implica la utilización de estas dos formas en distintos grados (Kocka y Haupt, 2009: 2-3). Para estos autores, la historia comparativa respondería a distintos pro-

\footnotetext{
8 Dado que existen distintas metodologías para abordar la historia transnacional, Struck, Ferris y Revel (2011: 576) han llegado a considerar dicha perspectiva como una suerte de "paraguas", que cobija diversas formas de ejercer la disciplina.

9 Otro enfoque que puede ser tenido en cuenta es el de la "historia conectada". Sin embargo, gran parte de sus presupuestos son compartidos por los diversos enfoques que hemos analizado en el texto. Subrahmanyam (1997) y Wakabayashi (2018).
} 
pósitos metodológicos. En términos heurísticos, "permitiría identificar problemas y preguntas que de otra manera resultarían imposibles de abordar". En términos descriptivos "ayudaría a clarificar las características de casos individuales". En términos analíticos "ayudaría a cotejar generalizaciones". Finalmente, en términos paradigmáticos, ayudaría a "desfamiliarizarse con lo familiar", ayudando de este modo a "reconsiderar las nociones de excepcionalidad de las explicaciones históricas propias" (Kocka y Haupt, 2009: 3-5).

Las características recién expuestas están presentes en los enfoques comparativos que realizan prácticamente todas las ciencias sociales. Sin embargo, según Kocka y Haupt, existen ciertas características específicas de la historiografía que imponen algunos desafíos especiales a dichos enfoques (Kocka y Haupt, 2009: 12-14). La primera de ellas sería el afán de la historiografía de apegarse a las fuentes, principalmente primarias, como criterio de validación. Esto implicaría que, a medida que se incluyen más casos de estudio en un estudio comparativo, la posibilidad de recurrir a las fuentes primarias para abordarlo disminuye. La segunda característica sería el énfasis que pone la disciplina histórica en el cambio a través del tiempo, lo que impediría que una "secuencia de casos sirva para ejemplificar leyes generales" (Kocka y Haupt, 2009: 13). De esta manera, el ejercicio comparativo de carácter sincrónico dificultaría la narración de la transformación a lo largo del tiempo. Finalmente, la tercera característica es que los historiadores "asumen que los componentes individuales de la realidad no pueden entenderse fuera de su relación con otros componentes de esta", lo que implicaría que "el aislamiento de variables sea menos factible y más limitado en la historia que en la economía o en la investigación social empírica" (Kocka y Haupt, 2009: 13). Por este motivo, en su práctica disciplinar, los historiadores comparatistas se verían obligados a desestimar muchos elementos contextuales, limitando su capacidad de trabajar con explicaciones multicausales (Kocka y Haupt, 2009: 14).

La historia comparativa ha sufrido importantes cambios desde la década de 1990, los que han implicado una transformación en sus métodos y enfoques de trabajo. En primer lugar, al énfasis en instituciones, procesos y estructuras sociales como objetos de estudio, que caracterizó a la historia comparada europea, especialmente alemana, 
entre las décadas de 1960 y 1980, se ha sumado en los últimos años la preocupación por aspectos culturales. En segundo lugar, se ha ampliado el foco de atención al momento de demarcar los límites de las unidades de comparación, las que tradicionalmente habían sido de carácter nacional. En la actualidad, sin dejar de reconocer la importancia de las fronteras nacionales, los estudios han tendido también a incluir ámbitos locales y regionales e incluso transnacionales (Kocka y Haupt, 2009: 17-19).

Como han sostenido Mónica Juenja y Margrit Pernau, "el problema de las relaciones existentes entre las unidades a ser comparadas se ha convertido en un importante debate en el campo de la historia comparativa durante los años recientes" (Juenja y Pernau, 2009: 109). En este sentido, según las autoras, "la necesidad de contextualizar y complejizar los ejercicios comparativos habría estimulado los estudios de las transferencias y sus variadas recepciones" (Juenja y Pernau, 2009: 109). Así, el historiador francés Michel Espagne, uno de los autores pioneros en este debate, planteó que la "teoría de transferencia cultural se concibe como contribución a una corrección a la metodología de la historia cultural comparativa" (Espagne, 1994: 191). No es de extrañar que, desde mediados de la década de 1990, haya comenzado a producirse un debate en que "fueron desarrollados diversos conceptos: primero el concepto de transfer, luego el concepto de entangled history; posteriormente el concepto de histoire croisée y finalmente la conceptualización de una combinación entre la historia comparativa y la relations history" (Kaelble, 2009: 33). Si bien existe una gran cantidad de definiciones y variantes de estos enfoques metodológicos, todos ellos comparten el hecho de ser enfoques relacionales. Hacemos referencia a estos enfoques para conocer las definiciones y perspectivas existentes sobre el estudio de las transferencias, a la vez que para incorporar elementos y prevenciones propios de los enfoques de carácter relacional. Así, respecto al concepto de transfer o transferencia, Harmut Kaelble lo ha definido como:

"El proceso del cual las normas, las imágenes y representaciones de una cultura aparecen en otras a través de la transmisión de conceptos. Dichas transmisiones se originan tanto a través de migraciones, como de encuentros y de la lectura de textos de otras culturas" (Kaelble, 2009: 34). 
Dentro del grupo de enfoques relaciones también se encuentra la histoire croisée o historia cruzada. En un afán por hacer cognoscibles las complejidades del fenómeno, Werner y Zimmermann han planteado que el esquema de histoire croisée sería una crítica a los énfasis lineales y unilaterales que, en opinión de los autores, habrían evidenciado los estudios de transferencia. ${ }^{10}$ Werner y Zimmermann han analizado algunos de los problemas de los estudios comparativos y de transferencia, señalando puntos críticos de estos. A su juicio, los enfoques comparativos adolecerían del problema de ser una "operación cognitiva", que tendería a operar en "términos binarios", respondiendo a la búsqueda de "diferencias y similaridades". Sin embargo, en las ciencias sociales se aplicaría a "sujetos históricos que están situados en y constituidos por dimensiones múltiples e interpenetradas" (Werner y Zimmermann, 2006: 33). Por otra parte, en opinión de Werner y Zimmermann, los estudios de transferencia no se harían cargo de los problemas de reciprocidad y reversibilidad; además, presupondrían estabilidad en los puntos de partida y llegada, dando por establecidos los marcos de referencia y la invariabilidad de las categorías de análisis. En este sentido, hacen un llamado a complejizar los puntos de vista, historizando y problematizando marcos referenciales y categorías de análisis (Werner y Zimmermann, 2006: 31-33). ${ }^{11}$

Teniendo en cuenta estas prevenciones, consideramos que, al momento de realizar una conceptualización de la noción de transferencia política, es necesario tener en cuenta muchas de las críticas planteadas por los cultores de la histoire croisée. Esto pues, al definir la transferencia política como la migración, a través de las fronteras nacionales, de prácticas, representaciones e ideas políticas, además de modelos de políticas ya implementados, se vuelve indispensable tener en cuenta el dinamismo de los actores y escenarios involucrados, especialmente al

\footnotetext{
10 Varios de los problemas y limitaciones señalados por Werner y Zimmermann también han sido abordados recientemente por Conrad (2017: 41-43).

11 Como ha sintetizado Mariana Perry, la "propuesta" de Werner y Zimmerman "se basa en que - a diferencia de otras propuestas relacionales, como la historia comparada o los estudios de transferencia- enfatiza la existencia de una multiplicidad de posibles puntos de vista y las diferencias que resultan de lenguajes, categorizaciones, y conceptualizaciones, tradiciones y usos disciplinares, lo que añade otra dimensión de reflexión a este tipo de estudios. Asimismo, la historia cruzada presenta la oportunidad de explorar cuestiones más generales, tales como la escala, categorías de análisis, la relación entre diacronía y sincronía y los regímenes de historicidad y reflexividad" (Perry, 2016: 19).
} 
analizar el rol cumplido por los agentes intermediadores que ejercen dichos procesos.

Si bien la literatura existente sobre los estudios de transferencia es extremadamente variada, consideramos que podemos distinguir tres tipos principales de modos de abordarla en las humanidades y las ciencias sociales. Estos son los estudios de transferencia cultural, los estudios de circulación de las ideas y, finalmente, los estudios de policy transfer o transferencia de políticas. Como veremos a continuación, algunos de estas vertientes se han desarrollado como compartimentos estancos en distintas disciplinas, con poca comunicación entre sí, pese a sus evidentes puntos de contacto y su potencial complementariedad. Lo que distinguiría a estos enfoques sería su diferente origen disciplinar - que los haría pertenecer a distintas tradiciones intelectuales-y, en menor medida, los tipos de agentes mediadores y los objetos de estudio en que centran su atención.

En primer lugar, respecto de los estudios de transferencia cultural, cabe destacar que corresponden a una tradición desarrollada por la historiografía, principalmente francesa y alemana. Al respecto, es necesario centrarse en los planteamientos realizados por uno de los investigadores pioneros en dicha área, Michel Espagne, quien ha sostenido que el estudio de la transferencia cultural aborda "el paso de los objetos culturales de un contexto a otro, dando como resultado la transformación de su significado, teniendo en cuenta los vectores que hicieron posible la transferencia" (Espagne, 2013: 1). Por este motivo, lo que distinguiría al estudio de las transferencias culturales del análisis de otros tipos de "intercambios culturales" sería el proceso de transformación del objeto cultural, el que sería entendible gracias al conocimiento de los agentes que la realizan y sus anclajes históricos específicos. Esta necesidad de conocer, contextualizar y hacer explícitos, en la narración historiográfica, a los agentes y características del proceso a través del cual realizan la transferencia, lleva a que, para el estudio de la transferencia cultural, no sea suficiente dar cuenta de las similitudes o simultaneidades culturales, y a la vez no se permita suponer la existencia de formas ocultas de vinculación (Ther, 2009: 208).

Los autores inscritos dentro de los estudios de transferencia cultural tienden a coincidir en cuanto a los objetos de la transferencia. Bajo el amplio rótulo de bienes culturales cabrían diversos elementos 
que podrían ser reinterpretables fuera de su contexto de origen, tales como ideas, prácticas o representaciones. Sin embargo, respecto de los agentes mediadores, tienden a existir énfasis discordantes. A tono con el ambiente contrario al nacionalismo metodológico, muchos autores han enfatizado en la autonomía del contexto nacional de dichos agentes. Así queda evidenciado en el programa de investigación planteado por Raquel Sánchez García, quien a su vez sigue los planteamientos de Jean Francois Botrel:

"En el análisis de la producción cultural resultaría más productivo hablar de relaciones asimétricas en las que cabe sugerir la hipótesis de que la dimensión nacional tiene tal vez menos significación que los contactos que se establecen entre otros núcleos del intercambio (círculos culturales, ciudades, movimientos, individuos)" (Sánchez García, 2016: 2).

Describiendo de una manera crítica estos enfoques, Henk Te Velde sostiene que "Dicha aproximación se ha concentrado exclusivamente en los contactos internacionales, especialmente en el ámbito de las ideas. Ella sostiene estudiar una comunidad cultural internacional y, por ende, puede alcanzar el estatus de una verdadera historia internacional" (Te Velde, 2005: 208).

Algunos autores, sin desconocer la potencial autonomía de otros núcleos de intercambio, han señalado la relevancia de lo nacional en el proceso de reinterpretación asociado a la transferencia. La importancia del rol activo de los agentes lleva a que autores como Philipp Ther hayan indicado que "al estudiar la transferencia no sólo sea necesario estudiar la aceptación y adaptación de ciertos bienes, sino también la exclusión y el rechazo deliberado de elementos culturales que fueron percibidos como foráneos" (Ther, 2009: 207). Por este motivo, la historia de las transferencias culturales implicaría poner atención a los ejercicios comparativos que los actores que estudiamos llevaron a cabo en su realidad: "El estudio de las transferencias culturales revela la historicidad de las comparaciones", así, quedaría claro cómo "la realización de comparaciones no sólo es un método abstracto, sino también una práctica histórica de larga data que causa transferencias culturales" (Ther, 2009: 208).

En segundo lugar, es necesario señalar las similitudes que presentan los estudios de transferencia cultural con otros enfoques similares, 
como son los estudios sobre circulación de las ideas. Respecto a estos cabe partir señalando la definición realizada por Eduardo Devés, a juicio de quien:

"La Circulación de las Ideas es el proceso de emisión y recepción de las ideas desde unas regiones hacia otras, asumiendo que en este transcurso se van produciendo mutaciones o hibridaciones y que en esta circulación hay diferentes 'estaciones', por una parte, así como diferentes 'especies', por otra" (Devés, 2004: 339).

Como queda en evidencia, tanto los enfoques de transferencia como los de circulación de las ideas abordan procesos de tránsito, teniendo en cuenta las transformaciones que el objeto de circulación sufre en su camino producto de la selectividad y la capacidad de reinterpretación que ejercen los agentes durante esta. A nuestro juicio, en este último caso la diferencia principal con los estudios de transferencia cultural radicaría en dos aspectos. En primer lugar, una diferencia de énfasis tiene que ver con el hecho de que los enfoques de circulación de las ideas, como dice su nombre, se centran en las ideas como su objeto principal, antes que en otros bienes culturales. En segundo lugar, una diferencia respecto al origen y las tradiciones disciplinarias se vincula con el hecho de que el esquema de circulación de las ideas proviene de la teoría literaria, y específicamente, dentro de ésta, de los estudios de teoría de la recepción, para luego pasar a ser adaptada por diversas disciplinas de las humanidades y las ciencias sociales, y, en el caso específico de la historiografía, por la subdisciplina de la historia intelectual. ${ }^{12}$

Los orígenes de dicho enfoque se derivan del afán de la teoría de la recepción por complejizar la historia del traspaso de ideas en distintos contextos, evitando simplificar dichos procesos en lo que Chris Bayly ha denominado como "categorías binarias de dominación y resistencia" (Bayly et al., 2006: 1454). Por el contrario, el enfoque de circulación de las ideas enfatizaría en la capacidad de los actores de apropiarse, adaptar y reinterpretarar las ideas. Al respecto, Pierre Bourdieu, en su

12 Sobre la Teoría de la Recepción véase el clásico texto de Robert C. Holub (2003). Una interesante descripción sobre la importancia de las teorías de la recepción y los esquemas de circulación de las ideas en la Historia Intelectual, puede ser encontrada en (Dotti et al., 2008: 11-19). 
clásico texto referido a las "Condiciones sociales de la circulación internacional de las ideas", llama a poner atención al hecho de que "los textos circulan sin su contexto" y que "no traen consigo el campo de producción del cual son productos", a la vez que "los receptores, quienes se encuentran en un campo diferente de producción, reinterpretan los textos de acuerdo con las estructuras de su campo de recepción" (Bourdieu, 2000: 221). Para luego concluir que:

"El sentido y la función de un trabajo [intelectual] foráneo no está simplemente determinado por su campo de origen, sino también por al menos en igual proporción el campo de recepción. Primero porque el sentido y función del campo original muchas veces son completamente desconocidos, pero también porque los procesos de transferencia de un campo doméstico a uno extranjero están hechos de una serie de operaciones sociales" (Bourdieu, 2000: 222).

Como puede desprenderse de los planteamientos de Bourdieu, el esquema de circulación de las ideas implica necesariamente un afán de poner atención a los agentes y mecanismos que hacen posible dicha circulación, teniendo en cuenta su historicidad.

En tercer lugar, respecto de los estudios de policy transfer o transferencia de políticas, estos hacen referencia, según la definición de Anneliese Dodds, al modo en que "las políticas públicas son transferidas de un país o jurisdicción a otra" (Dodds, 2012:249). Dichas reflexiones, que se han generado en el ámbito de la ciencia política y de la administración pública, hasta el momento no han tendido a ser mayormente considerados por la historiografía, pese a que sus aportes complementan muchos de los aspectos ya tratados.

Dodds distingue entre dos conceptos: el de policy transfer o transferencia de políticas, y el de policy learning o aprendizaje de políticas, los que se encontrarían profundamente relacionados entre sí. El primero ha sido definido como un proceso a través del cual "el conocimiento sobre cómo las políticas públicas, los arreglos administrativos, instituciones e ideas de un escenario político son utilizadas en el desarrollo del mismo tipo de instancias en otro escenario" (Dodds, 2012: 251). Este tipo de transferencia podría tener un carácter coercitivo cuando las políticas son impuestas mediante distintos mecanismos de presión, y no coercitivo cuando se toman decisiones de política pública simi- 
lares a los que se han adoptado en otros países, pero sin necesidad de que medie la fuerza u otro tipo de presiones en su adopción. El segundo dice relación con los intentos deliberados de adaptar al contexto local objetivos y técnicas de política pública de otros países. Esto implicaría que los tomadores de decisiones "estarían interesados en los desarrollos que tienen lugar en otros países, o en ejemplos que tuvieron lugar con anterioridad en el propio, con el fin de encontrar potenciales ejemplos de imitación o rechazo" (Dodds, 2012: 254). Así, según Dodds, la noción de aprendizaje de políticas sería más precisa que la de transferencia de política para identificar el modo en que los tomadores de decisiones generan contraejemplos o "aprendizaje de lecciones negativas”, según la conceptualización de Paul Cairney (2012: 257), en referencia al tipo de políticas públicas que no habría que seguir.

Creemos que estas nociones deben ser operacionalizadas en el marco de las investigaciones históricas, teniendo en cuenta los agentes y mecanismos que hicieron posible la transferencia. Esto, pues, no toda similitud entre las políticas públicas adoptadas por dos países respondería necesariamente a procesos de transferencia o aprendizaje, sino que también podría derivar del surgimiento de respuestas similares pero independientes a desafíos y problemas nacionales comunes o parecidos. Por estos motivos, Dodds advierte que, en muchos casos, "la existencia de procesos de transferencia de políticas termina por ser inferida antes que comprobada" (Dodds, 2012: 265-267).

Cairney ha insistido en la variedad de actores que pueden verse involucrados en estos procesos, que pueden ir desde organizaciones supranacionales y estados nacionales hasta organizaciones no gubernamentales y expertos en políticas públicas (Cairney, 2012: 263). De todas maneras, Dodds sostiene que hay ciertos elementos que condicionan la posibilidad de realizar un proceso de transferencia de políticas por parte de los actores. Entre los factores que facilitarían la transferencia se encontrarían, fundamentalmente, la proximidad, ya sea cultural, ideológica o geográfica, y, entre los elementos inhibidores destacarían las barreras lingüísticas e institucionales (Dodds, 2012: 268).

En esta misma lógica, y con un énfasis más normativo, Cairney, siguiendo los estudios de Dolowitz y Marsh (2000:17), explicita que el proceso de transferencia puede fallar al darse una "transferencia 
desinformada", es decir que el país que aplica la política cuente con información incompleta sobre ésta. Otra causa de falla en la transferencia puede deberse a su carácter incompleto, es decir, a que elementos relevantes de la política no fueron transferidos. Finalmente, se refiere a la categoría "transferencia inapropiada" para así nombrar los intentos de transferir una política sin tener en cuenta las condiciones contextuales adversas que dificultarían o imposibilitarían su implementación (Cairney, 2012: 259).

Como habíamos planteado, realizaremos nuestra conceptualización en el ámbito de los estudios de transferencia. Sin embargo, al hacerlo adoptaremos un enfoque que nos permita aunar elementos provenientes de los estudios sobre transferencia cultural, circulación de las ideas y transferencia de políticas. En este sentido, apuntaremos a crear una definición conceptual para el enfoque de trasferencia política, sosteniendo que dicho enfoque aborda la circulación, a través de las fronteras nacionales, de diversos elementos políticos como prácticas, representaciones e ideas políticas, además de modelos de políticas públicas ya implementados. Enfatizaremos el modo en que dicha transferencia se produce a través de agentes intermediadores, personales e indirectos, quienes transmiten la información sobre los elementos políticos, cuyos intereses e identidades están constreñidos por sus contextos de origen, pero pueden, eventualmente, independizarse y adquirir una autonomía relativa de estos, generando redes autónomas de transferencia. De igual manera, tendremos en cuenta el rol ejercido por los receptores en los procesos de transferencia política, considerando cómo estos adaptan y reinterpretan los elementos transferidos de acuerdo a sus intereses e identidades, pero también se ven constreñidos por las tradiciones y marcos institucionales imperantes, incluyendo los nacionales, reforzando así la posibilidad de inscribir dicho enfoque en el marco de la historia transnacional.

Así, los procesos de transferencia pueden tener finales diversos, que van desde la aceptación relativamente incondicional de los elementos transferidos hasta su rechazo, permitiendo el surgimiento de ejemplos a seguir o lecciones negativas. En nuestro esquema, la identificación de los agentes de la transferencia, incluyendo mediadores y receptores, y sus mutaciones y relaciones con marcos de referencia cambiantes, se vuelve fundamental, debido al protagonismo que tendrán tanto en 
la transmisión como en la transformación de los elementos políticos transferidos.

\section{El rol de los agentes en el proceso de la transferencia política}

Como habíamos señalado, uno de los elementos centrales a abordar, al tratar los procesos de transferencia política, es el rol jugado por los agentes. Al respecto, al estudiar la transferencia política se vuelve fundamental centrar la mirada en los actores que intervienen en el proceso de transferencia, identificándolos, distinguiendo sus tipologías, analizando su relación con el contexto en que se desenvuelven y los efectos que pueden llegar a producir, centrando la atención en la generación de modelos y contramodelos políticos. En este sentido, se vuelve necesaria la identificación de dos tipos principales de actores: los agentes intermediadores y los agentes receptores.

Parte del arsenal conceptual que desarrollaremos para abordar este punto está tomado de la literatura sobre las teorías de la difusión; dentro de esta, especialmente la referida a la difusión de formas de acción colectiva entre los movimientos sociales. ${ }^{13}$ Se trata de un campo de estudios con evidentes similitudes con el de los estudios de transferencia, entre los que, sin embargo, ha existido poco contacto. Dicho vacío ha venido a ser llenado por los trabajos de Henk Te Velde (2005: 208), quien ha establecido puentes entre ambas vertientes analíticas.

En primer lugar, distinguiremos los tipos de actores que actúan como agentes intermediadores. Entendemos intermediación como "la formación de vínculos (o la consolidación de los antiguos) entre transmisores y receptores" (Chabot y Duyvendak, 2002: 708). Al momento de establecer una distinción acerca de los distintos tipos de mediadores que ejercen los procesos de difusión, McAdam y Rucht han señalado que existirían dos modelos, "uno sería el relacional, el cual estaría caracterizado por los contactos directos e interpersonales", y otro sería el "no-relacional", determinado por la importancia de canales indirectos e impersonales de transmisión, como los medios de comunicación de masas (McAdam y Rucht, 1993: 59). Basándonos en su conceptua-

\footnotetext{
13 El trabajo clásico sobre teoría de la difusión, referido primordialmente a las innovaciones tecnológicas, es el de Everett Rogers (1995).
} 
lización, plantearemos que pueden existir dos tipos de formas de intermediación. En primer lugar, las formas directas de intermediación, definidas por la existencia de contactos personales, en los cuales el conocimiento entre los individuos jugaría un papel fundamental, y, en segundo lugar, las formas indirectas de intermediación, caracterizadas por su impersonalidad y su abstracción.

Respecto de las formas directas de intermediación, es necesario destacar que estas se encuentran marcadas por la existencia de contactos directos y personales entre individuos. Aunque dicha situación plantea un alto grado de dependencia de la iniciativa individual de los agentes, de todas maneras estos verían afectadas sus posibilidades por su capacidad de identificarse con los receptores y por la existencia de mecanismos institucionalizados de comunicación política. En efecto, para hacer viable su labor y erigirse como intermediadores, los agentes requerirían de un grado importante de identificación con los receptores. A dicha identificación se la ha denominado como "atribución de similaridad" (McAdam y Rucht, 1993: 59). La atribución de similaridad puede verse facilitada por la equivalencia institucional, es decir, la tendencia de organizaciones y actores a buscar sus contrapartes en los mismos tipos de instituciones existentes en otros países. Sin embargo, como han destacado McAdam y Rucht, la atribución de similaridad debe ser considerada como un "logro, el producto de una construcción social antes que una identificación automática” (McAdam y Rucht, 1993: 64). De un modo similar, la preexistencia de contactos institucionales entre actores políticos es un factor coadyuvante en el establecimiento de formas directas de mediación.

En segundo lugar, fuera de los contactos personales, se vuelve necesario poner atención a lo que hemos denominado como las "formas indirectas de intermediación". Como han sostenido Strang y Soule en sus investigaciones referidas a la difusión de las formas de acción colectiva, las "fuentes externas" de difusión, nombre que ellos asignan a este tipo de formas impersonales y abstractas, jugarían un rol relevante en el proceso. Dentro de las formas indirectas de intermediación, los medios de comunicación de masas tendrían un rol predominante. Reflexionando sobre el rol de los mass media, Strang y Soule sostienen que este no se reduciría al de un mero transmisor, sino que, también, ejercería una función amplificadora y editora (1998: 271). Así, instan- 
cias como la prensa juegan un papel relevante en la importancia que se le asigna al elemento transferido mediante su instalación en la agenda noticiosa, a la vez que participando de su edición, ya sea a través de su modificación, su cercenamiento o la alteración de las proporciones de alguno de sus componentes. ${ }^{14}$

Funciones similares son cumplidas por la industria editorial. Estas han sido abordadas por Pierre Bourdieu, quien se ha detenido a señalar las diversas "operaciones sociales" implicadas en la "transmisión" de un texto desde un campo doméstico a uno foráneo". Estas van desde el "etiquetamiento y clasificación" por parte de los editores, "la elección de la colección en la que se inserta", la redacción del "prefacio" o los estudios críticos - estos últimos, instancias privilegiadas para insertar el texto en el campo de recepción y explicarlo-, lo que permite al editor dar a conocer su punto de vista y "tomar una suerte de posesión de él" (Bourdieu, 2000: 222).

En todo caso, cabe señalar que la separación entre formas directas e indirectas de intermediación en las transferencias cumple una función más bien analítica, que ambas instancias se encuentran relacionadas y que tienden a mezclarse, ser complementarias y reforzarse entre sí (McAdam y Rucht, 1993: 59). Al respecto, se debe tener en cuenta que los contactos interpersonales influyen en las decisiones, tanto de la industria de los medios de comunicación como en la editorial. El mismo Bourdieu, en tono irónico señala las vinculaciones entre los contactos interpersonales y la industria editorial de las traducciones, analogándola a una "mafia del establishment", caracterizada por sus relaciones de reciprocidad (Bourdieu, 2000: 223).

Es dable destacar cómo los agentes mediadores pueden perpetuar su labor en el tiempo, llegando a constituir redes con ciertos grados de permanencia y con importantes niveles de autonomía respecto de sus lugares de origen. Se trata de un punto que ha sido abordado por estudiosos de diversas corrientes, incluyendo los estudios de difusión, de transferencia cultural y de circulación de las ideas. Diana Mishkova, quien ha estudiado procesos de transferencia política en los Balcanes decimonónicos, plantea que, en ciertos casos, tras el paso del tiem-

14 Sobre la capacidad de los medios de comunicación de instalar una agenda noticio- 
po, "no se puede identificar un patrón claro de transferencia -quién está innovando, quién transmite y quién recibe", esto pues se habría generado "una red internacional que ha desarrollado sus propias reglas" (Mishkova, 2012: 671). En esta situación influyen, como sostienen Chabot y Duyvendank, estudiosos de la difusión de las formas de acción colectiva, los esfuerzos puestos por los actores en la "construcción deliberada y concreta de redes transfronterizas entre individuos, grupos, organizaciones y países" (Chabot y Duyvendak, 2002: 699). En esta misma lógica, la historiografía ha tomado elementos desarrollados por los estudios sobre circulación de las ideas. Así, según Isabel Hofmeyr, "la preocupación por el 'transnacionalismo' habría implicado poner atención a 'los espacios [o paisajes] de circulación,' rescatando el término desarrollado por Arjun Appadurai, cuyo trabajo de los años 80 tardíos ha sido fundamental en el surgimiento de estas aproximaciones" (Bayly et al., 2006: 1444), ${ }^{15}$ al igual que los aportes de Manuel Castells (1999: 295). ${ }^{16}$

En este sentido, entenderemos los "espacios de circulación" como los conjuntos de redes de interacción, de carácter tanto institucional como interpersonal y mediático, que permiten la transmisión de los elementos políticos más allá de las fronteras y que han generado identidades e intereses propios, logrando ciertos niveles de autonomía respecto de las entidades que dieron origen a sus miembros.

En relación con los agentes receptores, es necesario indicar que su rol se encuentra marcado por el contexto en que estos realizan su la-

\footnotetext{
15 Según la interpretación de Kokot, un elemento a rescatar del pensamiento de Appadurai dice relación con cómo "diferentes tipos de actores" actúan como los "nodos en una red, concentrando gracias a su práctica cultural los flujos globales que pasan a través de sus lazos" (Kokot, 2006: 7). Dentro de los distintos paisajes de circulación, cabe mencionar especialmente al "etnopaisaje", concepto utilizado por Appadurai para referirse a al "paisaje de personas que constituyen el mundo cambiante en que vivimos: turistas, inmigrantes, refugiados, exiliados, trabajadores inmigrantes y otros grupos móviles y personas que constituyen una característica esencial del mundo y aparecen afectando las política de y entre los países en un grado sin precedentes", afectando las políticas entre y al interior de los Estados nacionales (Appadurai, 1990: 297).

16 Castells, en una definición pensada para ser operativa en el contexto de los actuales procesos de globalización, ha entendido a los espacios de circulación como los "arreglos materiales que permiten la simultaneidad de las prácticas sociales sin contigüidad territorial”. Añadiendo que “...el espacio de circulación está hecho de redes de interacción, y los fines y tareas de cada red configuran diferentes espacios de circulación" (Castells, 1999: 364).
} 
bor. De todas maneras, consideramos que los receptores no tienen un rol pasivo, sino que, por el contrario, cumplen un rol activo, adaptando y reinterpretando los elementos transferidos de acuerdo con sus intereses e identidades. Sin embargo, identidades e intereses se encuentran constreñidos, tanto por las tradiciones como por los marcos institucionales en que están inmersos.

Sobre la "tradición", indicaremos que dicho concepto se encuentra fuertemente asociado al de "identidad". Entendemos la tradición desde un punto de vista dinámico, y tenemos en cuenta, como ha sostenido Hobsbawm (1983), que muchas veces esta corresponde a intentos "inventados" de generar elementos de continuidad con el pasado, que responden a los afanes de "estructurar algunas partes de la vida social como si esta fuera inmutable e invariable" (2). Sin embargo, dicha reflexión no nos lleva a desestimar su importancia operativa. Por el contrario, adherimos a la conceptualización de Slobodan Naumovic, citada por Mishkova (2012), quien la ha definido "no como el segmento pasivo de un sistema que previene el cambio, sino como un conjunto de hábitos, memorias, normas y valores que, en vista de su continuidad, juegan un rol clave en el autoentendimiento de un grupo social", añadiendo que "la tradición funciona como un medio útil para legitimar las intenciones reales o ficticias de los actores políticos", y que "su uso" en muchos casos "depende más de los intereses de quienes lo instrumentalizan que en lugar de sus propias propiedades intrínsecas" (Mishkova, 2012: 670).

En relación con los marcos institucionales, consideramos los aportes realizados por los estudios institucionalistas históricos de la ciencia política, los que plantean que "las elecciones que se toman cuando se está formando una institución o cuando se formula una política tienen un efecto restrictivo en el futuro" (Greener, 2005: 62). Al respecto, desde la ciencia política se han adoptado modelos provenientes de la economía y, especialmente dentro de esta, de los estudios de las trayectorias tecnológicas. Estos han sostenido que, al igual que en el caso de la tecnología, "la política implica elementos propios del azar", tales como la "agencia" individual y las "elecciones"; "sin embargo, una vez que se elige un 'camino,' este puede quedar 'bloqueado,' coartando otras opciones para los actores, haciendo que estos ajusten sus estrategias para acomodarse al patrón predominante” (Thelen, 1999: 385). Así 
se entiende cómo los intereses de los actores están ligados y, en parte, definidos por los marcos institucionales en que se encuentran insertos.

Aportes más recientes, desde el punto de vista de la sociología institucionalista, han insistido en que el carácter de construcción social que tendrían las instituciones implica que estas responden a "marcos interpretativos" comunes o "cogniciones compartidas", lo que lleva a que la creación de nuevas instituciones tienda al isomorfismo", volviéndose compatible con las anteriores. Esto involucraría que, "incluso cuando los creadores de políticas proponen rediseñar las instituciones, se vean limitados por los marcos ya existentes" (Thelen, 1999: 386). De este modo, se evidencia de qué modo los marcos institucionales no solo influyen en los intereses de los actores, sino incluso en su cosmovisión y su identidad: es decir, en los esquemas interpretativos de su realidad.

Si bien estos esquemas han recibido críticas, en cuanto dificultarían el estudio del cambio político, remarcando de manera excesiva los elementos de estabilidad, deben ser tenidos en cuenta, ya que remarcan los límites que las instituciones ponen al desenvolvimiento y al entendimiento de los actores. Así, podemos entender que incluso sectores que no se acomoden a las políticas imperantes o deseen subvertirlas, ven limitados sus objetivos y estrategias por los arreglos institucionales imperantes. Es en este aspecto que consideramos relevante, siguiendo los planteamientos de Collier y Collier, poner atención a las "coyunturas críticas" - o momentos de "elecciones cruciales" - que pasarían a definir los límites de las arenas políticas (Collier y Collier, 1991: 27-29).

Por estos motivos, los procesos de transferencia no deben llevar al investigador a extremar el cosmopolitismo metodológico, ni a subestimar la importancia de los Estados nacionales en su análisis. Por el contrario, en momentos históricos y contextos geográficos, en los que el Estado nacional ha sido una institución hegemónica, este debe ser considerado como un factor operante en los procesos de transferencia. Por lo demás, el estudio de esta última puede ser de utilidad para conocer sus límites y fortalezas, a la vez que para enriquecer su historia. En este aspecto coincidimos con Diana Mishkova, quien plantea que los estudios de transferencia política "no abandonan la nación, sino que buscan complementar, desestabilizar y, por ende, afinar el análisis de los marcos nacionales" (Mishkova, 2012: 670). 
En el marco de estos constreñimientos podemos entender cómo los agentes receptores reinterpretan y transforman los elementos transferidos, generando una gama de actitudes que puede ir desde una apropiación casi completa hasta el rechazo radical. Esta visión, sin negar las asimetrías de poder y los efectos de la hegemonía cultural, logra superar los modelos más simplistas, centrados en las lógicas binarias de "dominación/imposición" o "sumisión/complicidad", para pasar a tener en cuenta las dinámicas de "apropiación" (French, 2003: 375). En efecto, como han sostenido Strang y Soule, tras la mayoría de los procesos de apropiación subyacería un "proceso interpretativo autoconsciente", el que respondería a los esquemas interpretativos" de los agentes receptores (Strang y Soule, 1998: 276).

\section{Reflexiones finales}

La noción de transferencia política permite estudiar fenómenos que trascienden las fronteras nacionales en contextos en los cuales los Estados nacionales son entidades operantes relevantes, habilitando al investigador para comprender de una manera más acabada la manera en que se generan modelos y contramodelos políticos. En este sentido, conviene situar la noción de transferencia en el marco del enfoque de la historia transnacional, antes que en el de la historia global. Esto pues aborda fenómenos de conexión y circulación en un marco en que es necesario tener en cuenta la capacidad de constreñimiento de los actores ejercida por los Estados nacionales, y, de manera especial, porque no se enfoca necesariamente en el estudio de fenómenos cuyo análisis haga inteligibles procesos globalizadores.

El esquema propuesto es tributario, fundamentalmente, de los estudios de transferencia cultural. Sin embargo, la incorporación de las críticas y prevenciones tomadas de otros enfoques relacionales en el marco de la historia transnacional, cómo la histoire croisée, además de los aportes de los estudios sobre circulación de las ideas y recepción, policy transfer y sociología de la difusión, permiten que el estudio de la transferencia política se complejice. Este afán facilita la generación de puntos de encuentro entre perspectivas y enfoques disciplinares con importantes elementos en común, los que, si bien presentan un potencial complementario, se han mantenido como compartimentos estancos con escaso grado de contacto. Los recién mencionados aportes nos ayudan a 
evitar la adopción de esquemas lineales, teniendo en cuenta la variedad de tipos de agentes involucrados en el proceso de transferencia y el hecho de que estos pueden encontrarse relacionados a través de vínculos de carácter más o menos estable. De la misma manera, permite entender que los agentes de la transferencia ejercen una apropiación crítica de los elementos que recepcionan, dentro del marco de constreñimientos dado por las instituciones y tradiciones en que están insertos.

Al tener en cuenta la capacidad de apropiación crítica, podemos sostener que los procesos de transferencia política pueden llevar a la generación de modelos, entendidos como ejemplos a seguir, o de contramodelos, es decir, lecciones negativas. Coincidimos con Henk Te Velde (2005) cuando plantea que lo que vuelve relevante el estudio de la transferencia política es su capacidad de "modificar la naturaleza de la política en el país receptor". Esto en cuanto uno de los principales intereses en el estudio de la transferencia está puesto en entender cómo "lo internacional" moldea en parte la política "nacional". Sin embargo, dicha "modificación" o moldeamiento no necesariamente implica un cambio radical. Las distintas reacciones de los receptores pueden llevar desde la adhesión a modelos creados fuera de las fronteras nacionales por los actores internos, reforzando las tendencias cosmopolitas de la política, hasta un rechazo que exalta la diferencia, reafirmando las tendencias nacionalistas y excepcionalistas de la política al interior de un Estado nacional.

\section{Referencias bibliográficas}

\section{a) Artículos y capítulos de libros}

Appadurai, A. (1990). "Disjuncture and Difference in Global Cultural Economy", en Theory, Culture and Society, N7, pp. 295-310.

Balachandran, G. (2015). "Transnational Histories and Subcontinental Pasts: A Review Essay", en The Indian Economic and Social History Review, vol. 22, ํ4, pp. 533-545.

Bayly, C. A., S. Beckert, M. Connelly, I. Hofmeyr, W. Kozol y P. Seed. (2006). "AHR Conversation: On Transnational History", en The American Historical Review, vol. 111, ํ5, pp. 1441-1464.

Bordieu, P. (2000). "The Social Conditions of the International Circulation of Ideas", en R. Shusterman, Bourdieu: A Critical Reader. Oxford: Blackwell, pp. 220-228. 
Cairney, P. (2012). “Policy Transfer", en P. Cairney, Understanding Public Policy. Theories and Issues. Basingstoke: Palgrave Macmillan, pp. 244-264.

Castells, M. (1999). “Grasrooting the Spaces of Flow", en Urban Geography, vol. 20, ํ4, pp. 294-302.

Chabot, S. y J. W. Duyvendak. (2002). "Globalization and the transnational diffusion between social movements: Reconceptualizing the dissemination of the Gandhian repertoire and the 'coming out' routine", en Theory and Society, vol. 31, №6, pp. 697-740.

Chatterjee, P. (2001). "Subaltern History", en N. J. Smelser, y P. B. Bates, International Encyclopedia of the Social \& Behavioral Sciences. Amsterdam: Elsevier, pp. 15237-15241.

Chernilo, D. (2006). "Socials's Theory Methodological Nationalism. Myth and Reality", en European Journal of Social Theory, vol. 9, $\mathrm{N}^{\circ} 1$, pp. 5-22.

Collier, R. B. y Collier, D. (1991). "Framework: Critical Junctures and Historical Legacies”, en R. B. Collier, y D. Collier, Shaping the Political Arena. Critical Junctures, the Labor Movement and Regime Dinamics in Latin America. New Jersey: Princeton University Press, pp. 27-39.

Conrad, S. (2009). "Double Marginalization: A Plea for a Transnational Perspective on German History", en J. Kocka y H. G. Haupt (Eds.), Comparative and transnational History. Central European Approaches and New Perspectives. New York-Oxford: Berghahn Books, pp. 52-76.

De Vries, J. (2103). "Reflections on Doing Global History", en M. Berg, Writing The History of the Global. Oxford: Oxford University Press, pp. 32-47.

Devés, E. (2004). "La circulación de las ideas y la inserción de los cientistas económico-sociales chilenos en las redes conosureñas durante los largos 1960", en Historia, vol. 37, ํ2, pp. 337-366.

Dodds, A. (2012). "Policy transfer and learning”, en A. Doods, Comparative Public Policy. Basingstoke: Palgrave Macmillan.

Dolowitz, D. y D. Marsh. (2000). "Learning From Abroad: The Role of Policy Transfer in Contemporary Policy Making", en Governance, vol. 13, $\mathrm{N}^{\circ} 1$, pp. 5-24.

Dotti, J., A. Blanco, M. Plotkin y L. García. (2008). "Encuesta sobre el concepto de recepción", en Políticas de la memoria. Revista de Investigación e información del CeDInCI, N8/9, pp. 1-19. 
Espagne, M. (1994). "Sur les limites du comparatisme en histoire culturel", en Geneses, vol. 17, N1, pp. 112-121.

Espagne, M. (2013). "La notion de transfert culturel", en Revue Sciences/ Lettres, 1 | 2013, http://rsl.revues.org/219, revisado el 22-I2017.

Fernández Abara, J. (2005). "Las raíces profundas del nacionalismo", en Ciencias Sociales Online, vol. 2, $\mathrm{N}^{\circ} 1$, pp. 75-81.

French, J. (2003). "Diasporic Dialogue, and the Errors of Pierre Bourdeiu and Loïc Wacquant", en Nepantla: Views from South, vol. 4, $\mathrm{N}^{\circ} 2$, pp. 375-389.

Greener, I. (2005). "State of the Art: The Potential of Path Dependence in Political Studies", en Politics, vol. 25, N 1, pp. 62-72.

Hobsbawm, E. (1983). "Introduction: Inventing Traditions", en E. Hobsbawm y T. Ranger, The Invention of Tradition. Cambridge: Cambridge University Press, pp. 1-14.

Juenja, M. y M. Pernau. (2009). "Lost in translation? Trascending Boundaires in Comparative History", en H. G. Haupt y J. Kocka (eds.), Comparative and transnational History. Central European Approaches and New Perspectives. New YorkOxford: Berghahn Books, pp. 105-129.

Kaelble, H. (2009). "Between Comparisons and Transfers -and What Now? A French-German Debate", en H. G. Haupt y J. Kocka (eds.), Comparative and transnational History. Central European Approaches and New Perspectives. New York-Oxford: Berghahn Books, pp. 33-38.

Kocka. J, y H. G. Haupt. (2009). "Comparison and Beyond. Traditions, Scope, and Perspectives of Comparative History", en H. G. Haupt y J. Kocka (eds.), Comparative and transnational History. Central European Approaches and New Perspectives. New York-Oxford: Berghahn Books, pp. 1-30.

Maldonado-Torres N. (2007). "Sobre la colonialidad del ser: contribuciones al desarrollo de un concepto", en S. Castro-Gómez y R. Grosfoguel, El giro decolonial. Reflexiones para una diversidad epistémica más allá del capitalismo global. Bogotá: Iesco-Pensar-Siglo del Hombre Editores, pp. 127-167.

Mazlish, B. (1993). "An Introduction to Global History", en B. Mazlish y R. Buultjens, Conceptualizing Global History. Boulder-San Francisco-Oxford: Westview Press, p. 1-26. 
McAdam, D. y D. Rucht. (1993). "The Cross National Diffusion of Movement Ideas", en The Annals of American Academy of Political and Social Science, vol. 528, $\mathrm{N}^{\circ} 1$, pp. 56-74.

McCombs, M. E. y D. L. Shaw. (1972). “The agenda-setting function of mass media", en Public Opinion Quarterly, vol. 36, №2, pp. 176-187.

Mishkova, D. (2012). "Liberalism and Tradition in the Nineteenth-Century Balkans: Toward History and Methodology of Political Transfer", en East European Politics and Societies and Cultures, vol. 26, Nº, pp. 668-692.

Osterhammel, J. (2009). A “'Transnational' History of Society: Continuity or New Departure", en H. G. Haupt y J. Kocka (eds.), Comparative and transnational History. Central European Approaches and New Perspectives. New York-Oxford: Berghahn Books, pp. 39-51.

Sánchez García, R. (2016). "Circulación de ideas y transferencias culturales: Espina y Europa en el siglo XIX", en Hispania Nova. Revista de Historia Contemporánea, $\mathrm{N}^{\circ} 14$, pp. 265-268.

Strang, D. y S. Soule. (1998). "Diffusion in Organizations and Social Movements: From Hybrid Corn to Poison Pills", en Annual Review of Sociology, vol. 24, $\mathrm{N}^{\circ} 1$, pp. 265-290.

Struck, B, K. Ferris y J. Revel. (2011). "Introduction: Space and Scale in Transnational History", en The International History Review, vol. 33, Nº 4, pp. 573-585.

Subrahmanyam, S. (1997). "Connected Histories. Notes Towards a Reconfiguration of Early Modern Eurasia", en Modern Asian Studies. vol. 31, Nº3, pp. 735-762.

Te Velde, H. (2005). “Political transfer: An Introduction”, en European Review of History, vol. 12, ํ2, pp. 205-221.

Thelen, K. (1999). "Historical institutionalism in Comparative Politics", en Annual Review of Political Science, $\mathrm{N}^{\circ}$ 2, pp. 369-404.

Ther, P. (2009). "Comparisons, Cultural Transfers and the Study of Networks: Towards a transnational history of Europe", en H. G. Haupt y J. Kocka (eds.), Comparative and transnational History. Central European Approaches and New Perspectives. New York-Oxford: Berghahn Books, pp. 204-225.

Wakabayashi, J. (2018). “Connected history and histoire croisée”, en L. D’Hulst y Y. Gambier (eds.), A History of Modern Translation 
Knowledge: sources, concepts and effects. Amsterdam, Philadelphia: John Benjamins Publishing Company, pp. 261-265.

Werner, M. y B. Zimmerman. (2006). "Beyond Comparison. Histoire Croisée and The Challenge of Reflexivity", en History and Theory, vol. 45, $\mathrm{N}^{\circ} 1$, pp. 30-50.

b) Libros

Anderson, B. (1983). Imagined Communities: Reflections on the Origin and Spread of Nationalism. London: Verso.

Burke, P. (1999). La revolución historiográfica francesa. Barcelona: Gedisa.

Conrad, S. (2017). What is Global History? Princeton and Oxford: Princeton University Press.

Chernilo, D. (2007). A Social Theory of the Nation State. The Political Forms of Modernity Beyond Methodological Nationalism. London: Routledge.

Gellner, E. (1983). Nations and nationalism. Ithaca: Cornell University Press.

Hastings, A. (1997). The Construction of Nationhood. Ethnicity, Religions and Nationalism. Cambridge: Cambridge University Press.

Hunt, L. (2014). Writing history in the global era. New York: W.W. Norton.

Hobsbawm, E. (1992). Nations and nationalism since 1780. Cambridge: Cambridge University Press.

Holub, R. C. (2003). Reception Theory. New York: Routledge.

Iggers, G. (2012). La historiografía del siglo XX. Santiago, Chile: Fondo de Cultura Económica.

Mignolo, W. (1995). The Darker Side of the Renaissance: Literacy, Territoriality, and Colonization. Ann Arbor: University of Michigan Press.

Osterhammel, J. y Petersson, N.P. (2019). Breve historia de la globalizción. Del 1500 a nuestros días. Buenos Aires: Siglo Veintiuno Editores.

Rogers, E. M. (1995). Diffusion of Innovations. New York: Free Press.

Smith, A. (2008). Nationalism: Theory, Ideology, History. Cambridge: Polity. 


\section{c) Ponencias}

Baeza, A. (2015). Global History and Latin America. Nature of Connections. En Symposium, School of Modern Languages. Congreso llevado a cabo en la Universidad de Bristol, Bristol, Inglaterra.

Kokot, W. (2006). Culture and Space-anthropological. En Swiss Graduate Programme en Ethnology/Anthropology. Key Concepts in Social Anthropology. Module Workshop Space Congreso llevado a cabo en Basilea, Suiza.

d) Tesis

Mantra, R. (2010). Speaking Subalterns: A Comparative Study of African American and Dalit/Indian Literatures. A dissertation submitted in partial fulfillment of the requirements for the Degree of Doctor of Philosophy. Tampa: Department of English. College of Arts and Sciences. University of South Florida.

Perry, M. A. (2016). La dimensión internacional del pensamiento político chileno. Aprendizaje y transferencia en el exilio. Proefschrift ter verkrijging van de graad van Doctor aan de Universiteit Leiden. Leiden: Instituut voor Geschiedenis, Universiteit Leiden. 